\title{
Exposing undergraduate engineering students to nonlinear differential equations using a practical approach in project based learning environments
}

\section{Dr. Günter Bischof, Joanneum University of Applied Sciences}

Throughout his career, Dr. Günter Bischof has combined his interest in science and engineering application. He studied physics at the University of Vienna, Austria, and acquired industry experience as development engineer at Siemens Corporation. Currently he is an associate professor at Joanneum University of Applied Sciences and teaches engineering and applied mathematics.

\section{Mr. Maximilian Brauchart,}

Maximilian Brauchart is currently studying Automotive Engineering at the Joanneum University of Applied Sciences. Prior to his studies, he attended a grammar school in Graz.

\section{Mr. Patrick Jenni,}

Patrick Jenni is currently studying Automotive Engineering at the University of Applied Sciences JOANNEUM Graz. After completing his studies, he intends to work in development.

\section{Mr. Jeremias Pirker,}

Jeremias Pirker is currently studying Automotive Engineering at the University of Aplied Sciences Joanneum Graz. After completion of his studies, he would like to participate in the development of technical innovations.

\section{Mr. Julian Sachslehner}

Julian Sachslehner is currently studying Automotive Engineering at the University of Applied Sciences Joanneum Graz. Before he started his studies he worked as a car mechanic and gained some practical experience in the automotive sector.

\section{Mr. Christian J. Steinmann,}

Christian Steinmann has an engineer degree in mathematics from the Technical University Graz, where he focused on software quality and software development process assessment and improvement. He is manager of HMS IT-Consulting and provides services for SPiCE/ISO 15504 and CMMI for development as certified Automotive SPiCE assessor. He performed more than 100 process assessments in software development departments for different companies in the finance, insurance, research, automotive, and automation sector. Currently, his main occupation is a consulting project for process improvement for safety related software development in the automobile sector. On Fridays, he is teaching computer science introductory and programming courses at Joanneum University of Applied Sciences in Graz, Austria.

\section{Mr. Tobias Markus Zörweg,}

Tobias Markus Zörweg is currently studying Automotive Engineering at the University of Applied Sciences Joanneum Graz. After completion of his studies, he aspires to work in development. 


\title{
Exposing undergraduate engineering students to nonlinear differential equations using a practical approach in project-based learning environments
}

\begin{abstract}
The increasing complexity inherent in the technologies currently being developed has profound implications for engineering education. Our students have to face increasingly complex adaptive systems organized in recursive, nonlinear feedback loops. A more pronounced attention to nonlinear systems could thus enable our students to understand not only the usefulness but also the limits of linearity, and facilitate the comprehension of more complex systems. This is especially true for mathematics, as differential equations play a fundamental role in the modeling and analysis of complex systems.
\end{abstract}

Courses in ordinary differential equations are part of the majority of undergraduate engineering curricula, and typically cover analytical solution methods for first-order and linear higher-order differential equations, as well as an introduction to numerical methods like the explicit Euler integration. Increasingly, nonlinear differential equations are included in the curricula, with a focus on phase portraits, stability, and local linearization, based on standard systems like the predator-prey equations.

The present work introduces an application of nonlinear differential equations in a classical engineering problem that is well-suited for undergraduate education, namely the optimal design of a dynamic vibration absorber. Such absorbers are mounted in a wide variety of structures to reduce the amplitude of mechanical vibrations, and are frequently designed as coupled spring-mass-damper systems. Linear absorbers can be tuned to suppress the resonance frequency of the structure, but unfortunately only within a relatively small frequency bandwidth. The introduction of nonlinearity into the damper system can overcome that drawback and increase the attenuation bandwidth. A perpendicular spring configuration, for instance, introduces a nonlinearity of the Duffing type and can increase the absorber's effective bandwidth significantly.

The mathematical modelling and numerical simulation of such a nonlinear absorber was carried out within the framework of a multidisciplinary undergraduate student project. The integration of the coupled system of differential equations was programmed in C\#, with a graphical user interface that provides a display of the vibrating system, a graph of the mass displacements over time, the system's response function, phase-space diagrams, and Poincaré sections. The adjustable parameters of the vibration absorber can be modified interactively during the simulation, which facilitates the identification of parameter sets leading to instabilities that can intensify the system's vibration instead of suppressing it. The dynamic visual output of the program is expected to increase the students' understanding of tuned vibration absorbers and of the transition of nonlinear dynamic systems into chaotic states.

In this paper, the theoretical background, the approach to the problem and the outcome of the undergraduate student project are presented and discussed. 


\section{Introduction}

The twenty-first century is widely considered to be an age of transformation, which presents us with challenges of a new dimension, scale and scope. The environment for engineering practice is changing fast and irreversibly, impelled by the impact of rapidly expanding information technology, the globalization of markets and manufacturing, and the imperatives of environmental protection and sustainable development. The complexity inherent in the newest technologies and their interaction with society has created the demand for a new approach to undergraduate engineering education. At the end of the past century, the National Science Foundation issued a program announcement for an action agenda for a systemic engineering education reform [1]. The program pursued the strategy of a paradigmatic shift in engineering education, characterized by active, project-based learning, by introducing mathematical and scientific concepts in the context of application, and by integrating the development of teamwork, communication, and problem-solving skills in learning experiences throughout the engineering curriculum. An engineering education based on this vision should produce graduates better prepared to meet the needs of engineering employers and increase student motivation and interest [2].

To accomplish such a shift in undergraduate engineering education, efforts towards a holistic engineering curriculum that is compatible with the complexity inherent in the technologies currently being developed have been encouraged (see, e. g., [3]). The key distinction drawn here is between engineered systems that are "complicated" (industrial age technologies of the $20^{\text {th }}$ century) and those that are "complex" (post-industrial age technologies of the $21^{\text {st }}$ century). The context within which industrial age technologies are understood, and on which traditional undergraduate engineering education is based, is a reductionist, deterministic and dualistic worldview. Post-industrial age technologies, on the other hand, are frequently characterized by a certain degree of complexity, uncertainty and ambiguity. Complex systems can have properties that are only exhibited by the whole and hence cannot be described in a reductionist way. They can show chaotic behavior and, in addition, some aspects may not be describable by any objective means at all.

A contextual shift from a linear reductionist mindset to a nonlinear holistic mindset is proposed for contemporary undergraduate engineering education [3]. The authors recognize that many of the core engineering and science courses that lay the foundation of the traditional engineering education are taught from a linear context. However, students should understand not only the usefulness but also the limits of linearity, which serves as the basis for a better comprehension of complex systems. The authors advocate the use of computer codes for solving nonlinear problems in order to help the students understand the underlying physical and mathematical concepts of nonlinear systems and networks.

The proposed shift from a reductionist to a holistic paradigm involves not only new context and content, but also a corresponding pedagogical concept; a project-based learning and team learning approach is suggested in [1]-[3]. Project assignments should be formulated in such a way that the students are encouraged to work on them through independent research, both individually and in teams. This would provide students with practical real-world experiences early on in their education and with a useful training in participatory skills.

At the Institute of Automotive Engineering at Joanneum University of Applied Sciences we have implemented project-based learning (PBL) environments since around the turn of the century [4]. During the last two decades a coherent procedure has been established as part of the undergraduate curriculum, which familiarizes students in a competitive way with the demands of contemporary industry. The students are confronted, complementary to their regular courses, with problems that are of a multidisciplinary nature and demand an appropriate level of technical proficiency. The project assignments are formulated in such a 
way that students are encouraged to work on them through independent research, which gives the students the chance to look beyond the standard curriculum of engineering education.

The first phase of this multi-subject PBL encompasses the second semester in the curriculum and is based on the Information Systems and Programming course. In this one-year course the programming language $\mathrm{C \#}$ is introduced, which allows the development of graphical user interfaces with comparatively little effort. In addition, it is an object-oriented programming language based on $\mathrm{C}++$ and thus enables the programming of fast and machine-oriented algorithms.

The project tasks are usually defined by members of the faculty and sometimes by industrial partners in such a way that students are requested to apply knowledge (already available or still to acquire) of informatics, mathematics, science and engineering to appropriate engineering problems. When the scope of the project is deemed feasible for the timeframe of one semester, it is ready to be added to a list of project proposals. The final results of all projects in this first PBL phase are stand-alone C\# software programs that can be installed and run on MS Windows operating systems.

The second phase of our multi-subject PBL is starting in the sophomore year and not connected with any particular subject. The students are encouraged to define research and development projects they would like to work on. One of the major projects is Formula SAE [5], an international competition for students of several engineering disciplines. This competition provides the students with a real-life exercise in design and manufacturing in a highly competitive context. The students are supposed to develop, design, build, test and put into international competition a formula-style race car within one year of work.

The remainder of this paper is structured by first providing a brief introduction to the initial phase of our multidisciplinary project-based learning procedure. Secondly, the principle of operation of linear and nonlinear dynamic vibration absorbers is described. Then the implementation into student-written computer programs is discussed and the programs are exemplified by numerical simulations.

\section{Dynamic vibration absorbers within the framework of multi-subject student projects}

The dynamic vibration absorber project was just one in a varied list of project proposals offered to the students. This list comprised project tasks like the visualization of Fourier transformations, a regenerative braking simulation, an n-body simulation for a virtual moon flight, the programming of chase and escape computer games using collective intelligence, an analyzing software for real-world driving emission measurements, and another dynamic vibration absorber using Coriolis force for a pendulum type system.

In accordance with our PBL procedure, the students were first introduced to all the projects at a kick-off meeting with the supervisors early in the semester. The supervisors presented the project tasks they have created and delivered detailed information on the scope of the projects, the deliverables, the timetable and deadlines, and the evaluation criteria. A one page task description of each project was then displayed and time allotted for project groups to form and make their selections. In order to foster a certain element of competition, which often increases the students' motivation, two or more groups could select a particular task. After initial consultation with the supervisors and the assignment of duties within the team, the students went through the following stages: researching topics, acquiring relevant background information and skills, finding mathematical solutions, designing and programming the software, and documenting the project progression and final result. The role of the supervisors was to guide the students through these stages and to give them advice and support for a successful completion of the projects [4]. At the end of the semester, each team was required 
to hand in the computer program and the project documentation. Both the software and the documentation were evaluated and assessed by the supervisors. On the last day of the semester, every project was presented to all the other students of the class and the subject supervisors. Students and supervisors alike evaluated the presentations based on predefined criteria, and winning teams were chosen.

The numerical simulation of dynamic vibration absorbers represents a typical project assignment for student teams within the framework of our first phase of multi-subject PBL. Dynamic vibration absorbers, also known as tuned-mass dampers, have become a popular means of mitigating vibration-induced damage since the German naval architect Hermann Frahm applied for a patent on the invention of such a device in 1909 [6]. They are mounted in structures to reduce the amplitude of mechanical vibrations in order to prevent structural failure, damage, or even just discomfort. Typical fields of applications include vibration absorption in seismic or aerodynamic excitation of structures, wind-induced flutter vibrations in power transmission lines, and torsional vibrations in rotating and reciprocating machines.

In its simplest implementation a tuned-mass damper consists of an absorber mass elastically coupled to the host structure. Systems of elastically coupled masses are typically covered in undergraduate mathematics and mechanics courses, and the modelling and simulation of such a system is therefore well-suited for student project assignments. The students know the behavior of an externally excited mass-spring-damper system, and have an idea of the energy transfer in coupled mass-spring systems. The fact, however, that an undamped dynamic vibration absorber can prevent an undamped primary mass from a resonance catastrophe, provides them with new and astonishing insight. From the students' point of view, this project has the advantage that they can start on firm ground before they delve deeper into the matter.

An even deeper immersion into numerical simulations of dynamic systems is possible when nonlinearity emerges. As distinguished from systems where the nonlinearity represents an undesired effect, tuned-mass damper can be intentionally designed to exploit nonlinear behavior. One of the main problems of linear tuned-mass dampers is the significant drop in effectiveness for small deviations from the resonance frequency. Nonlinear techniques can help increase the absorber bandwidth.

Due to the fact that our undergraduate mathematics curriculum has been tailored to the needs of the science and engineering courses in the wide spectrum of topics in automotive engineering (see the Appendix in [7]), the mathematical methods needed for the numerical solution of nonlinear differential equations are provided to the students already in the second semester of study. The Engineering Mathematics courses in the second and third semester of undergraduate study follow typically the contents of textbooks like Kreyszig's "Advanced Engineering Mathematics" [8], with some emphasis on numerical methods. The second semester course comprises linear algebra, ordinary differential equations, coupled systems of ordinary differential equations, numerical methods for the solution of ordinary differential equations and Laplace transformations. Hence, all the mathematical methods for the analytical solution of linear coupled systems of differential equations with constant coefficients, and for the numerical solution of arbitrary nonlinear differential equations are taught within the project period. The circumstance that the mathematical methods necessary for the solution of their projects are provided more or less simultaneously increases the students' attentiveness immensely and often leads to an improved class participation and interaction during the lectures [9].

With all the ingredients at hand, three teams of three students each were ready to accept the challenge to write a computer program for the numerical simulation and visualization of dynamic vibration absorbers. Only minimum requirements were defined in the project assignments and no limits were placed on the students' creativity or on the amount of time 
they should invest in order to complete the projects. This kind of creative freedom paired with the competition between the teams led to one acceptable and two very presentable results. All the figures presented in this paper are based on the programs written by those latter two teams, hereinafter referred to as "group A" and "group B".

\section{Equations of motion of dynamic vibration absorbers}

Dynamic vibration absorbers are widely used passive vibration control devices. They can be realized as a comparatively lightweight additional mass (the absorber mass) elastically coupled to the vibrating system that shall be stabilized against violent motion caused by harmonic excitation.

Figure 1 shows such a damped primary system (mass $m_{1}$, damping value $d_{1}$, and stiffness $c_{1}$ ) attached by a damped dynamic vibration absorber with the mass $m_{2}$, damping value $d_{2}$, and stiffness $c_{2}$, respectively. The excitation of the primary system is provided by $\boldsymbol{F}(t)$.

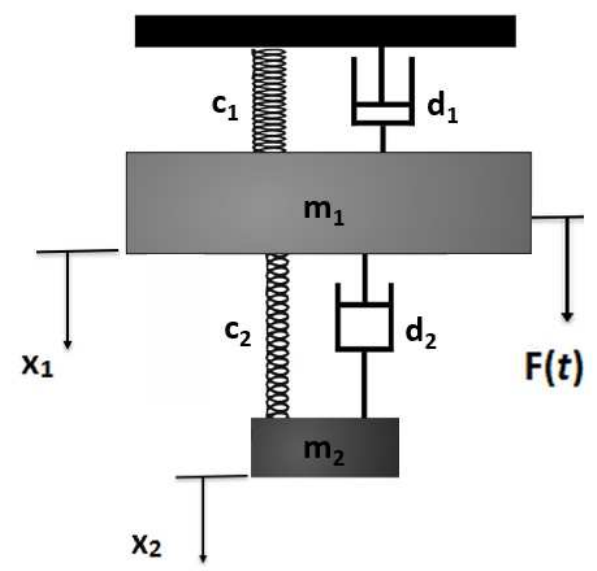

Figure 1: Dynamic vibration absorber

The equations of motion of the system in the case of harmonic excitation are

$$
\begin{aligned}
& m_{1} \ddot{x}_{1}+d_{1} \dot{x}_{1}+c_{1} x_{1}+d_{2}\left(\dot{x}_{1}-\dot{x}_{2}\right)+c_{2}\left(x_{1}-x_{2}\right)=F_{0} \sin (\omega t) \\
& m_{2} \ddot{x}_{2}+d_{2}\left(\dot{x}_{2}-\dot{x}_{1}\right)+c_{2}\left(x_{2}-x_{1}\right)=0
\end{aligned}
$$

with $x_{1}$ and $x_{2}$ being the displacements of the primary mass and the absorber mass, respectively.

In matrix notation, the equations of motion take the familiar form

$$
\mathbf{M} \ddot{\mathbf{x}}+\mathbf{D} \dot{\mathbf{x}}+\mathbf{C x}=\mathbf{F} \sin (\omega t)
$$

with the displacement vector $\mathbf{x}=\left(\begin{array}{c}x_{1}(t) \\ x_{2}(t)\end{array}\right)$, external excitation vector $\mathbf{F}=\left(\begin{array}{c}F_{0} \\ 0\end{array}\right)$, mass matrix $\mathbf{M}=\left(\begin{array}{cc}m_{1} & 0 \\ 0 & m_{2}\end{array}\right)$, damping matrix $\mathbf{D}=\left(\begin{array}{cc}d_{1}+d_{2} & -d_{2} \\ -d_{2} & d_{2}\end{array}\right)$, and stiffness matrix $\mathbf{C}=\left(\begin{array}{cc}c_{1}+c_{2} & -c_{2} \\ -c_{2} & c_{2}\end{array}\right)$.

The steady state response of the system will be of the form

$$
\mathbf{x}(t)=\mathbf{a} \cos (\omega t)+\mathbf{b} \sin (\omega t)
$$


so that Equation (3) can be solved analytically by substituting (4) into (3) and comparing the coefficients of the sine and cosine functions, yielding

$$
\left(\begin{array}{l}
\mathbf{a} \\
\mathbf{b}
\end{array}\right)=\left(\begin{array}{cc}
\mathbf{C}-\omega^{2} \mathbf{M} & \omega \mathbf{D} \\
-\omega \mathbf{D} & \mathbf{C}-\omega^{2} \mathbf{M}
\end{array}\right)^{-1}\left(\begin{array}{l}
\mathbf{0} \\
\mathbf{F}
\end{array}\right)
$$

An absorbing mass-spring system tuned to the resonance frequency of an undamped massspring system $(\mathbf{D}=\mathbf{0})$ reduces the motion of the main mass $x_{1}$ at this critical frequency to zero; the energy of the main mass is apparently totally absorbed by the dynamic vibration absorber. And although the undamped main mass would virtually experience a resonance catastrophe, the motion of the absorber is finite at this frequency, even without damping in both mass-spring systems. This is because the single-mass system has changed to a twodegree of freedom system with two resonance frequencies, with neither of them being equal to the original resonance frequency.

In the early 1930s, the Dutch-American mechanical engineer Jacob Pieter Den Hartog first tackled the optimum solution of a damped dynamic vibration absorber that is attached to an undamped primary system [10]. He found the optimum tuning parameter by utilizing the feature of fixed-point frequencies, i.e., frequencies, at which the response amplitude of the primary mass is independent of the absorber damping.

In the presence of damping in both mass-spring systems, however, the motion of the main mass will no longer be zero at the target frequency (see the red curve in Figure 2 at resonance frequency). The fixed-point feature is lost and a closed-form solution for optimum tuning parameters becomes impossible [11]. Real systems always possess a certain degree of damping and, in addition, dynamic vibration absorbers are frequently implemented with a hydraulic or frictional component in order to transform kinetic energy of vibration into heat.

Figure 2 shows the frequency response $s$ of the main mass $m_{1}$ as a function of normalized frequency, i.e., the driving frequency divided by the resonance frequency $\left(\omega / \omega_{c}\right)$ of the damped primary system. The blue curve shows the displacement of the primary system without absorber. The red curve represents the response function of the main mass after the tuned dynamic absorber $\left(m_{2} \approx 0.14 m_{1}\right)$ has been attached. Two new resonance frequencies have been created, located above and below the original resonance frequency.

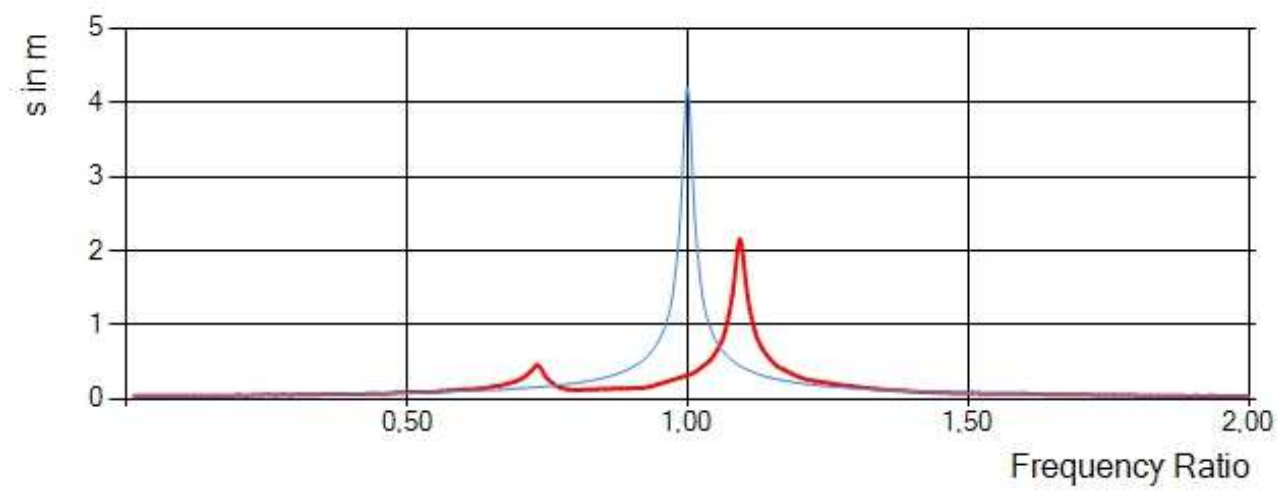

Figure 2: Primary mass displacement (blue: without absorber, red: with absorber)

The heights of the two new resonance peaks can be adjusted by changing the stiffness and damping of the tuned-mass damper, the split between the two peaks by altering the mass of the absorber. 


\section{The impact of nonlinearity on dynamic vibration absorbers}

Modern engineering increasingly tends to exploit nonlinear dynamical phenomena instead of avoiding them, as was commonly the case. Accordingly, nonlinearity is also utilized for vibration absorption and energy harvesting since it could be shown that nonlinear energy sinks can extract energy from virtually any mode of a host structure [12]. A typical example is the nonlinear tuned vibration absorber, which can absorb disturbances in wider ranges of frequency than linear tuned vibration absorbers due to its increased bandwidth [13]. A unique feature of this device is that it can enforce equal peaks in the frequency response of the coupled system for a large range of motion amplitudes, thereby generalizing Den Hartog's equal peak method to nonlinear systems [14].

An implementation of a certain degree of nonlinearity in tuned vibration absorbers can be achieved by simply attaching additional horizontal springs to the absorber mass (Figure 3 ).

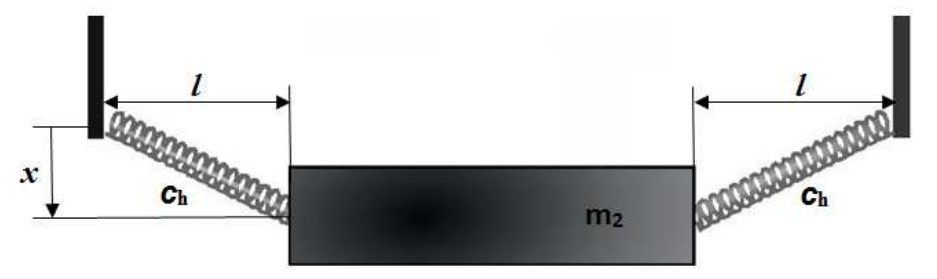

Figure 3: Additional horizontal springs attached to the absorber mass

When the absorber mass is displaced downwards by $x$, the restoring force $F_{\mathrm{h}}$ provided by the two horizontal springs in $x$ direction is given by

$$
F_{\mathrm{h}}=2\left(c_{\mathrm{h}}\left(\sqrt{l^{2}+x^{2}}-l\right)+F_{\mathrm{p}}\right) \frac{x}{\sqrt{l^{2}+x^{2}}}
$$

with $c_{\mathrm{h}}$ being the spring stiffness of the horizontal springs and $F_{\mathrm{p}}$ the preload of the springs in horizontal installation position [15]. The Maclaurin expansion of $F_{\mathrm{h}}$ with respect to $x$ yields

$$
F_{\mathrm{h}}=\frac{2 F_{p}}{l} x+\left(\frac{c_{h} l-F_{p}}{l^{3}}\right) x^{3}+O\left(x^{5}\right) .
$$

The first-order coefficient in Equation (7) yields together with the stiffness of the vertical spring $c_{2}$ the total linear spring stiffness $c_{2}^{\prime}$ of the absorber; the third-order coefficient $c_{n l}=\left(c_{h} l-F_{p}\right) / l^{3}$, on the other hand, determines the degree of nonlinearity in the system.

Figure 4 depicts the implementation of the above concept into a nonlinear tuned-mass damper system. The stiffness and damping in the system is once again represented by springs and dashpot elements.

The equations of motion of the modified system with third-order nonlinearity in the primary mass-absorber interaction are

$$
\begin{gathered}
m_{1} \ddot{x}_{1}+d_{1} \dot{x}_{1}+c_{1} x_{1}+d_{2}\left(\dot{x}_{1}-\dot{x}_{2}\right)+c_{2}^{\prime}\left(x_{1}-x_{2}\right)+c_{\mathrm{nl}}\left(x_{1}-x_{2}\right)^{3}=F_{0} \sin (\omega t) \\
m_{2} \ddot{x}_{2}+d_{2}\left(\dot{x}_{2}-\dot{x}_{1}\right)+c_{2}^{\prime}\left(x_{2}-x_{1}\right)+c_{\mathrm{nl}}\left(x_{2}-x_{1}\right)^{3}=0
\end{gathered}
$$


in the case of harmonic excitation. Equations (8) and (9) represent a modified form of the Duffing equation, which can lead to chaotic patterns of motion. The nonlinearity of $\left(x_{2}-x_{1}\right)$ is, in fact, a necessary condition for chaotic behavior, though not sufficient in itself [16].

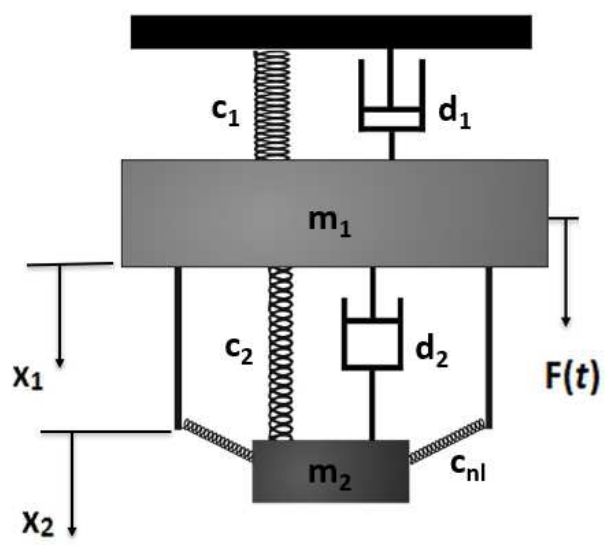

Figure 4: Nonlinear dynamic vibration absorber

As mentioned above, nonlinear absorbers can significantly increase the comparably small effective bandwidth of a simple tuned-mass absorber. On the other hand, however, it could be shown that nonlinearities can introduce instabilities that may increase unwanted vibration in the system instead of suppressing it [15]-[17]. Therefore, a deliberate choice of parameters is required for the design of a nonlinear absorber. Figure 5 shows the effect of the Duffing-like nonlinearity on the primary mass deflection amplitude $s$ for the same parameters as in Figure 2. A small third-order spring stiffness $\left(c_{\mathrm{nl}} \approx 0.12 c_{2}\right)$ reduces the higher resonance peak and shifts it away from the excitation frequency.

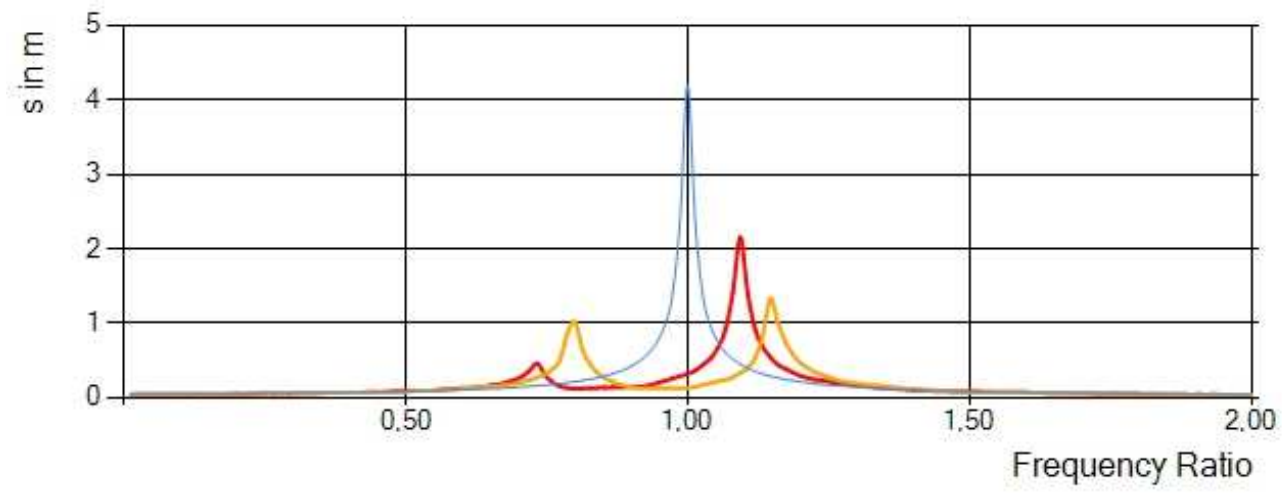

Figure 5: Primary mass displacement (red: linear absorber, yellow: nonlinear absorber)

\section{Implementation into computer programs}

The computer programs for the simulation of dynamic vibration absorbers of the types shown in Figures 1 and 4 were written by the student project teams in C\# within the object-oriented software development environment Visual Studio 2017. Both programs are organized in several subroutines, which comprise the numerical solution of the vibration absorber system's equation of motion, the readout and illustration of the results and the visualization of the motion of both primary and absorber mass.

Although closed form solutions to the equations of motion of the linear vibration absorber exist (see Equation (5)), it was decided to make use of numerical integration schemes for both the linear and the nonlinear dynamic vibration absorbers. For the solution to the equations of 
motion of e.g. the nonlinear tuned-mass damper (i.e., Equations (8) and (9)), the substitution $z_{1}=x_{1}, z_{2}=\dot{x}_{1}, z_{3}=x_{2}, z_{4}=\dot{x}_{2}, z_{5}=t$ is carried out. In this way the non-autonomous differential Equations (8) and (9) with the second time derivatives of $x_{1}$ and $x_{2}$ and an explicitly time-dependent right-hand side can be rewritten as the following system of nonlinear, autonomous first-order differential equations

$$
\begin{aligned}
& \dot{z}_{1}=z_{2} \\
& \dot{z}_{2}=\frac{1}{m_{1}}\left[F_{0} \sin \left(\omega z_{5}\right)-d_{1} z_{2}-c_{1} z_{1}+d_{2}\left(z_{4}-z_{2}\right)+c_{2}^{\prime}\left(z_{3}-z_{1}\right)+c_{\mathrm{nl}}\left(z_{3}-z_{1}\right)^{3}\right] \\
& \dot{z}_{3}=z_{4} \\
& \dot{z}_{4}=\frac{1}{m_{2}}\left[d_{2}\left(z_{2}-z_{4}\right)+c_{2}^{\prime}\left(z_{1}-z_{3}\right)+c_{\mathrm{nl}}\left(z_{1}-z_{3}\right)^{3}\right] \\
& \dot{z}_{5}=1
\end{aligned}
$$

The same set of equations can be used in the case of the linear vibration absorber by simply setting $c_{\mathrm{nl}}=0$. Such a set of equations $\dot{\mathbf{x}}=\mathbf{F}(\mathbf{x})$ can be easily solved numerically by means of algorithms taught in the accompanying Engineering Mathematics 2 course.

For the numerical integration of Equation (10) different numerical methods with varying orders of the truncation error have been implemented. Both project teams have offered the fourth-order Runge-Kutta (RK4), the explicit Euler and implicit Heun's method, which are all part of the mathematics course content. In addition, one group programmed the AdamsBashforth-Moulton and the Dormand-Prince method with adaptive stepsize control (see "Integrator" pull-down menu in Figure 8). Both methods are not subject of the standard engineering mathematics course. The latter is currently the default method in the ode 45 solver for MATLAB and GNU Octave and is the default choice for the Simulink's model explorer solver. Thus, different orders of accuracy can be tested and compared for the same time step size, an additional benefit when the program is used for instructional purposes.

The numerical integration provides $z_{1}=x_{1}, z_{2}=\dot{x}_{1}, z_{3}=x_{2}$, and $z_{4}=\dot{x}_{2}$ instantaneously for every time step, so that the displacements of primary mass and absorber mass over time and the phase portraits of both masses ( $\dot{x}_{1}$ over $x_{1}$ and $\dot{x}_{2}$ over $x_{2}$ ) can be plotted simultaneously. An additional advantage of the program is its ability to handle dynamic alterations to some input parameters, which affect the numerical results and the plots immediately (see slider bars in Figures 6 and 7).

Supplementary to the phase portraits the visualisation of Poincaré sections, i.e., a reduction of the dimensionality of the phase spaces of the masses by a stroboscopic registration of the system coordinates, is implemented in the program.

The input fields in the graphical user interfaces of both programs comprise all the parameters of Equations (8) and (9), the simulation time step and the simulation period. Figure 6 shows the second layer of the graphical user interface of the computer program designed by project group A. This window opens after the system parameters have been entered in the main menu and an integration method has been selected. In Figure 6 the primary and absorber mass deflections and velocities are displayed for both a linear and a nonlinear vibration absorber. In addition, the phase space diagrams for the primary mass, a Poincaré section and the frequency response of the primary mass are shown.

Since the programs are intended for demonstration purposes in education, particular effort was made for the visualization of the tuned-mass damper systems. For that reason both project 
groups have implemented the animated motion of both simple and nonlinear dynamic vibration absorbers.

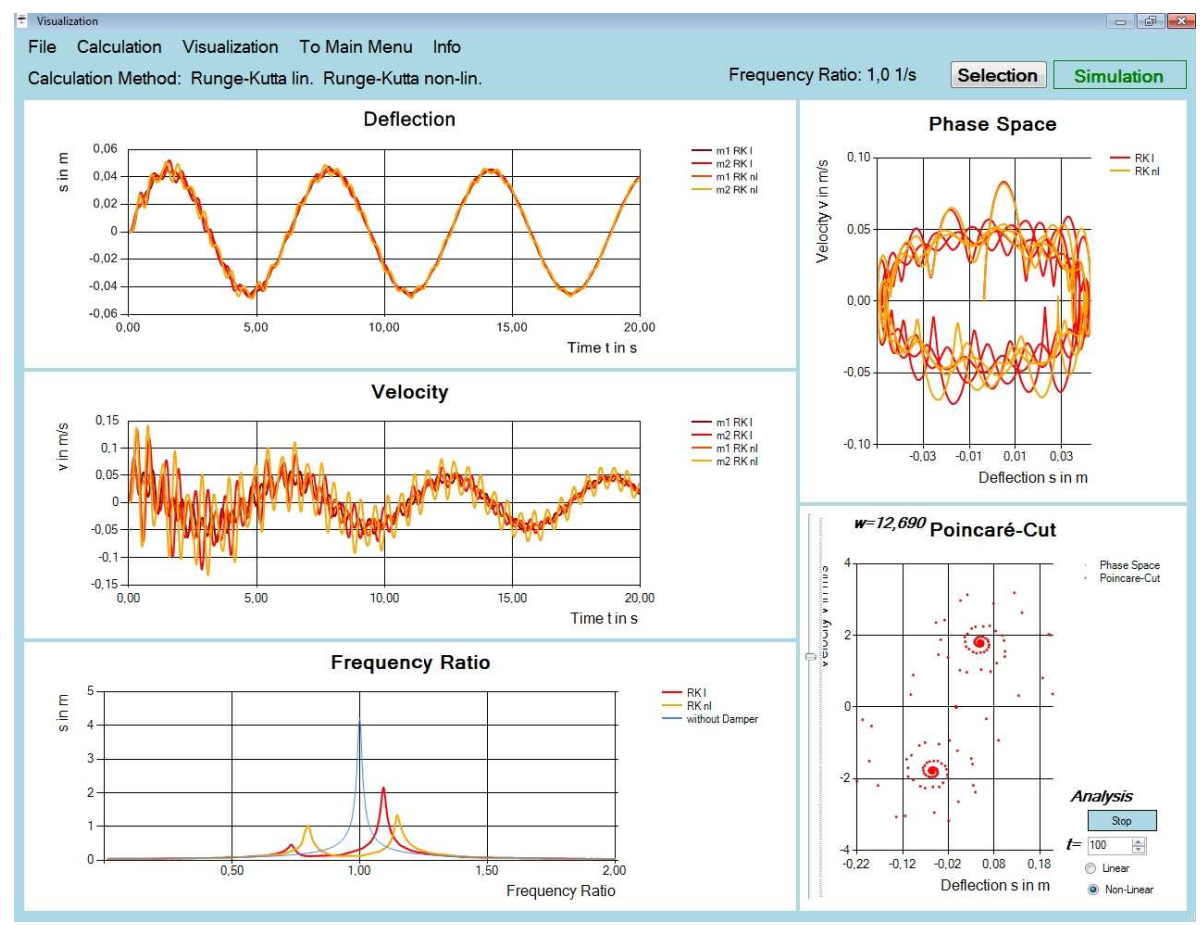

Figure 6: Graphical user interface of the computer program of project group A

In these animations, the masses move according to the results obtained from the numerical integration of Equation (10), and their associated springs and dashpots are correspondingly stretched and compressed.

In Figure 7 the motions of both a simple and a nonlinear absorber system are shown together with their deflection-time histories.

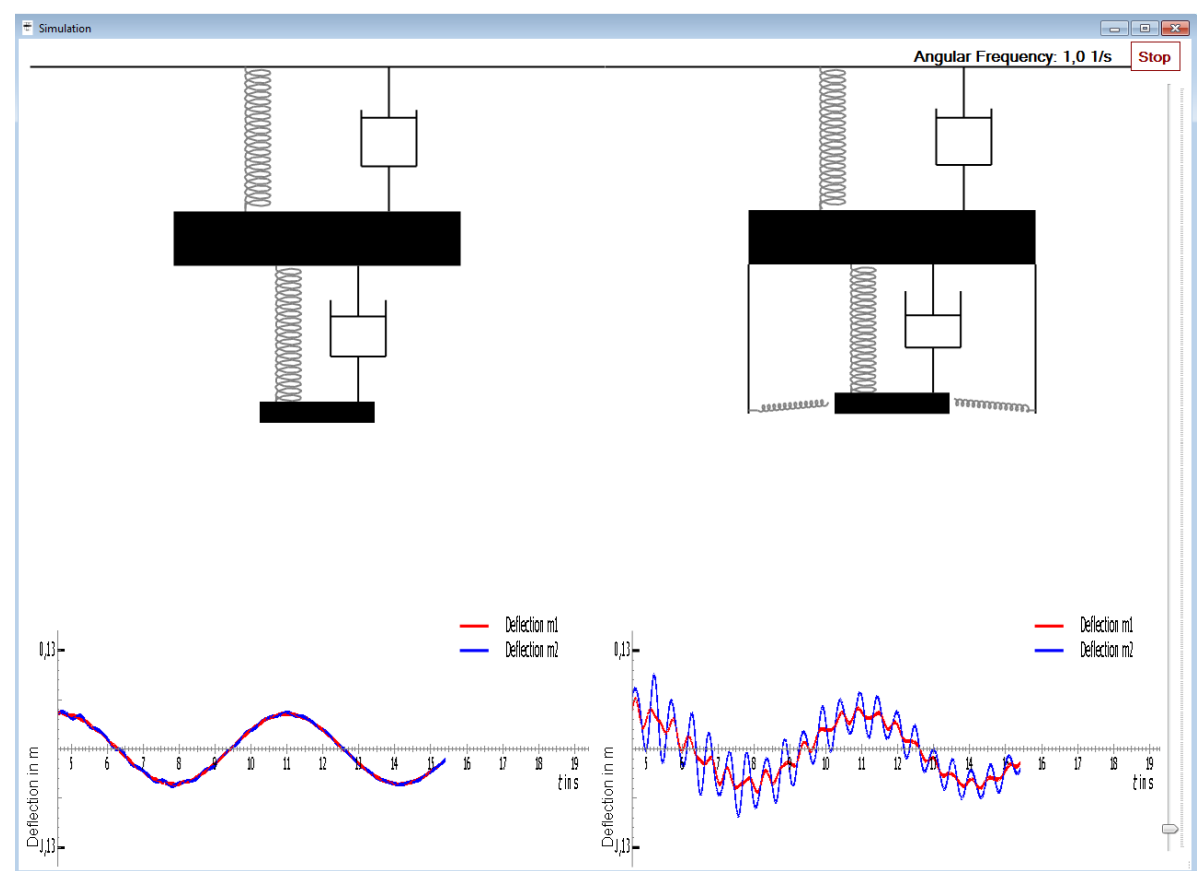

Figure 7: Joint visualization of the animated motion of both linear and nonlinear system (group A) 
The nonlinear absorber uses the same parameters as the linear one, with only a small nonlinearity added by the horizontal spring. This nonlinearity introduces a higher frequency vibration into the two-mass system that may smooth out when suitable system parameters are chosen.

Project group B plots a perspective projection of the animated vibration absorber system (Figure 8). The relative size of the masses adapts to the mass ratio, which made it reasonable for the group to place the parameter input and the motion animation in the same window. The frequency response diagram was moved to another tab due to the lack of available space (see Figure 9).

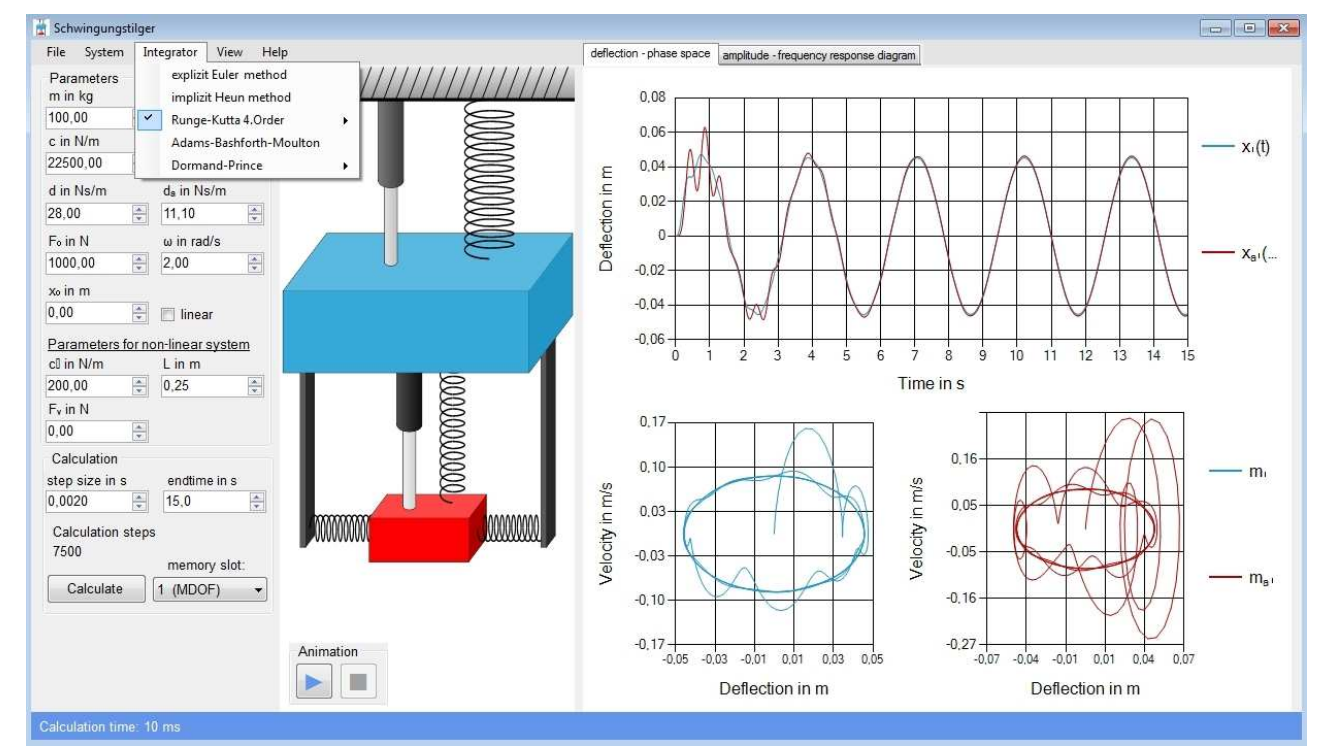

Figure 8: Graphical user interface of project group B's computer program

The program provides five memory slots that allow the simulation of up to five different configurations and a joint visualization of the simulation results.

A special feature of this group's solution is that the program enables the automatic calculation and visualization of a frequency sweep when using the RK4 integration method, which allows the illustration of the system's frequency response in the time domain.

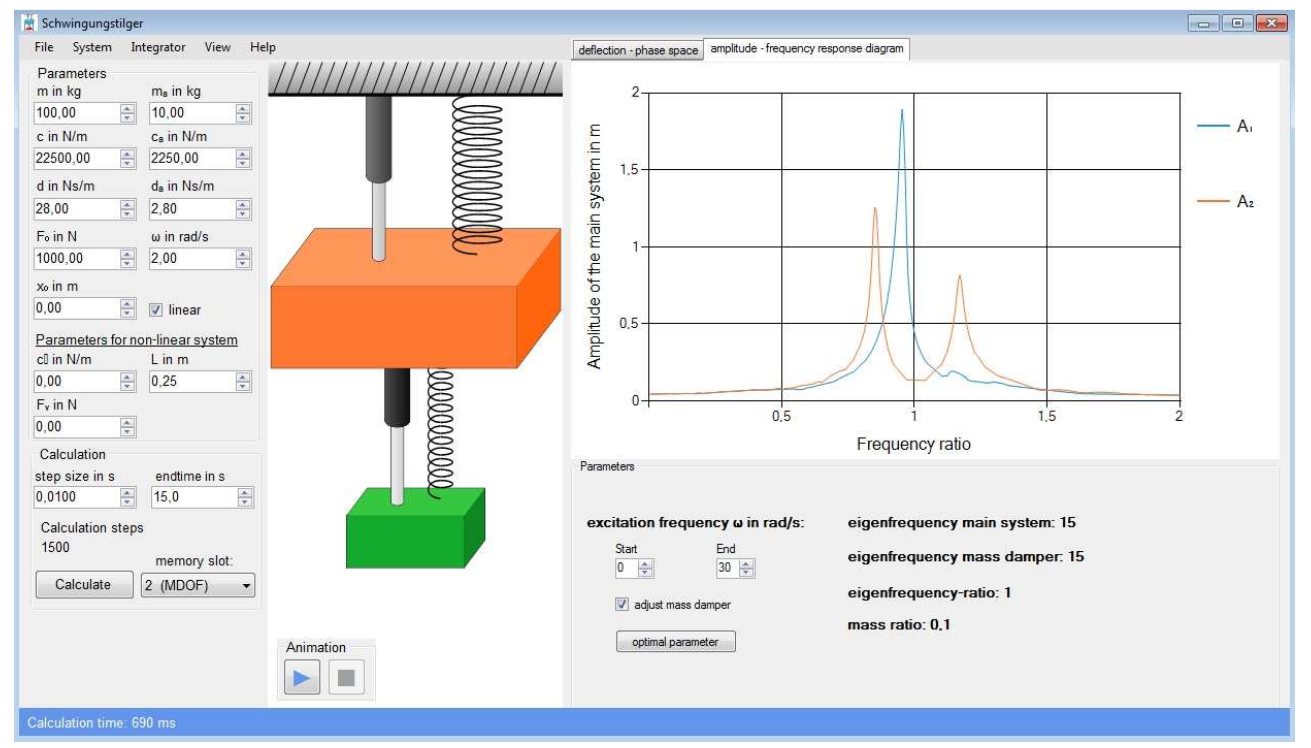

Figure 9: Second tab of project group B's graphical user interface 
The second tab comprises, in addition to the representation of the frequency response function, a parameter optimization calculator. The estimation of the optimal damper parameters follows Den Hartog's procedure for finding the optimal tuning parameters in undamped vibration absorbers [10], which is reasonable in the case of comparatively small damping constants. According to this procedure, the optimal ratio of absorber eigenfrequency $\left(\omega_{2}\right)$ to primary mass eigenfrequency $\left(\omega_{1}\right)$ is given by

$$
\frac{\omega_{2}}{\omega_{1}}=\frac{1}{1+m_{2} / m_{1}} .
$$

For a given mass ratio $\left(\mu=m_{2} / m_{1}\right)$, the absorber mass eigenfrequency $\omega_{2}$ can be adjusted by a proper choice of the absorber spring stiffness and damping constant.

\section{Numerical simulation of dynamic vibration absorbers}

The effect of a dynamic vibration absorber is numerically tested in group B's program for a spring mass system with a resonance angular frequency $\omega_{\mathrm{c}}=15 \mathrm{rad} / \mathrm{s}$. The system is excited at its critical frequency $\left(\omega=\omega_{c}\right)$, which leads to a continuous increase of the oscillation amplitude. Due to dissipative damping a maximum elongation is reached after some time (Figure 10 left).
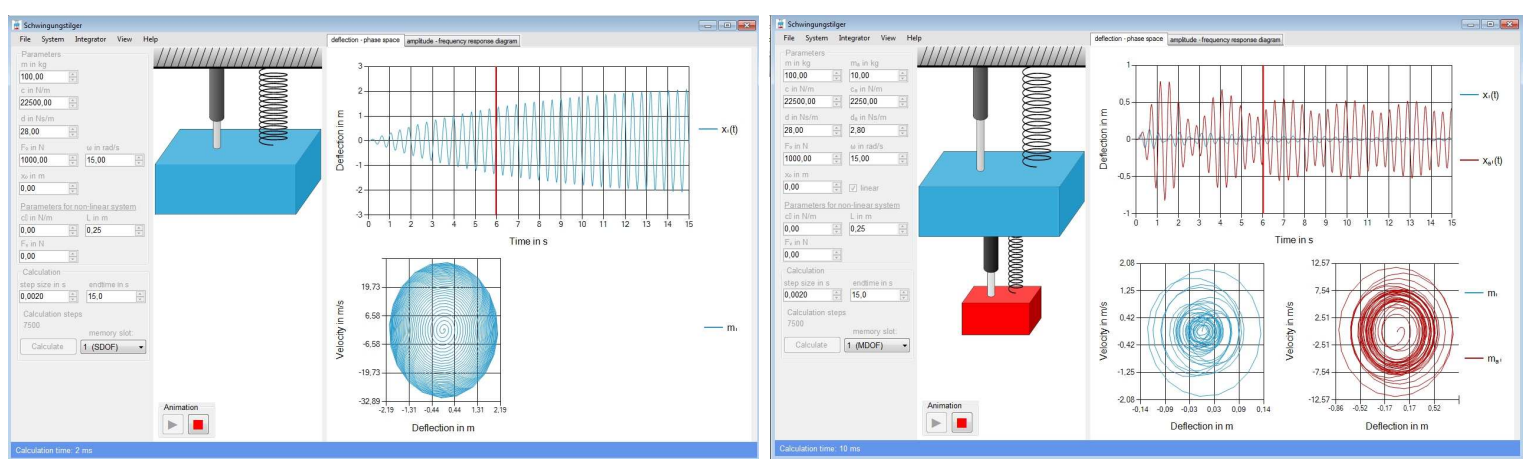

Figure 10: Critically excited oscillator $\left(\omega / \omega_{\mathrm{c}}=1\right)$ without (left) and with linear vibration absorber

The attachment of even a simple dynamic vibration absorber with $\mu=0.1$ and $\omega_{2} / \omega_{1}=1$ reduces the primary mass deflection considerably (Figure 10 right).

Further reduction of oscillation amplitude can be observed by employing a nonlinear vibration absorber. In the left part of Figure 11 the absorber parameters of the simple absorber in Figure 10 are used, with additional horizontal springs with $10 \%$ stiffness of the vertical absorber spring. In the right part of Figure 11 the nonlinear tuned-mass damper has been optimized according to Equation (11), which leads to a further reduction of oscillation amplitude and a faster approach of the phase portrait to a limit cycle.
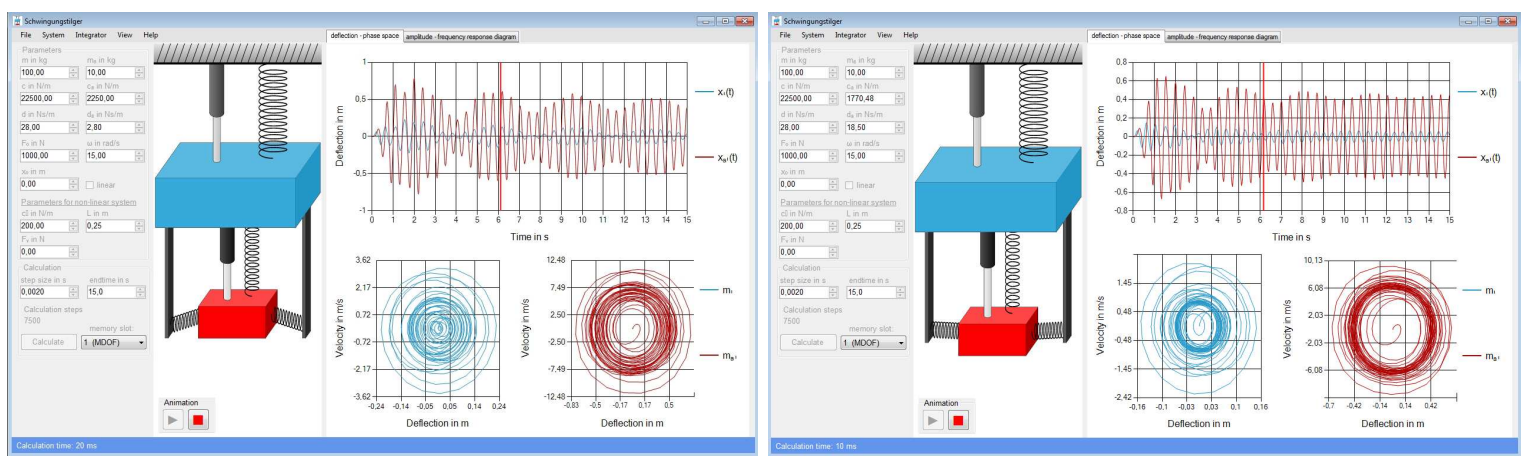

Figure 11: Comparison of not optimized (left) and optimized nonlinear vibration absorber $\left(\omega / \omega_{\mathrm{c}}=1\right)$ 
The inverse color code for primary and absorber mass was chosen by group A. The deflection time history of the primary mass is colored red and of the absorber mass blue, respectively. While the plot of the mass deflections over time acts like a sliding window that follows the current oscillation state of the system, the phase space diagram (in this program of the primary mass only) would become smeared out after some time due to damping or chaotic behavior. In order to avoid a totally reddened diagram, the trajectory fades to a pale orangered for values further in the past (see Figures 13-15).

In Figure 12 the effect of a linear and an optimized nonlinear absorber on the oscillating primary mass is compared in group A's program at an excitation frequency close to one of the two resonance frequencies of the coupled system. In the case of the linear absorber, the mass ratio $\left(m_{1} / m_{2}\right)$ and the spring stiffness ratio $\left(c_{1} / c_{2}\right)$ equal 10 . The primary mass as well as the spring stiffness keep unchanged in the case of the optimized nonlinear absorber, but the absorber mass is moderately increased. The absorber spring stiffness is reduced by about $10 \%$, while an additional stiffness of the same order is added by means of the horizontal springs. As a consequence, both primary and absorber mass deflections are markedly reduced in the nonlinear system.

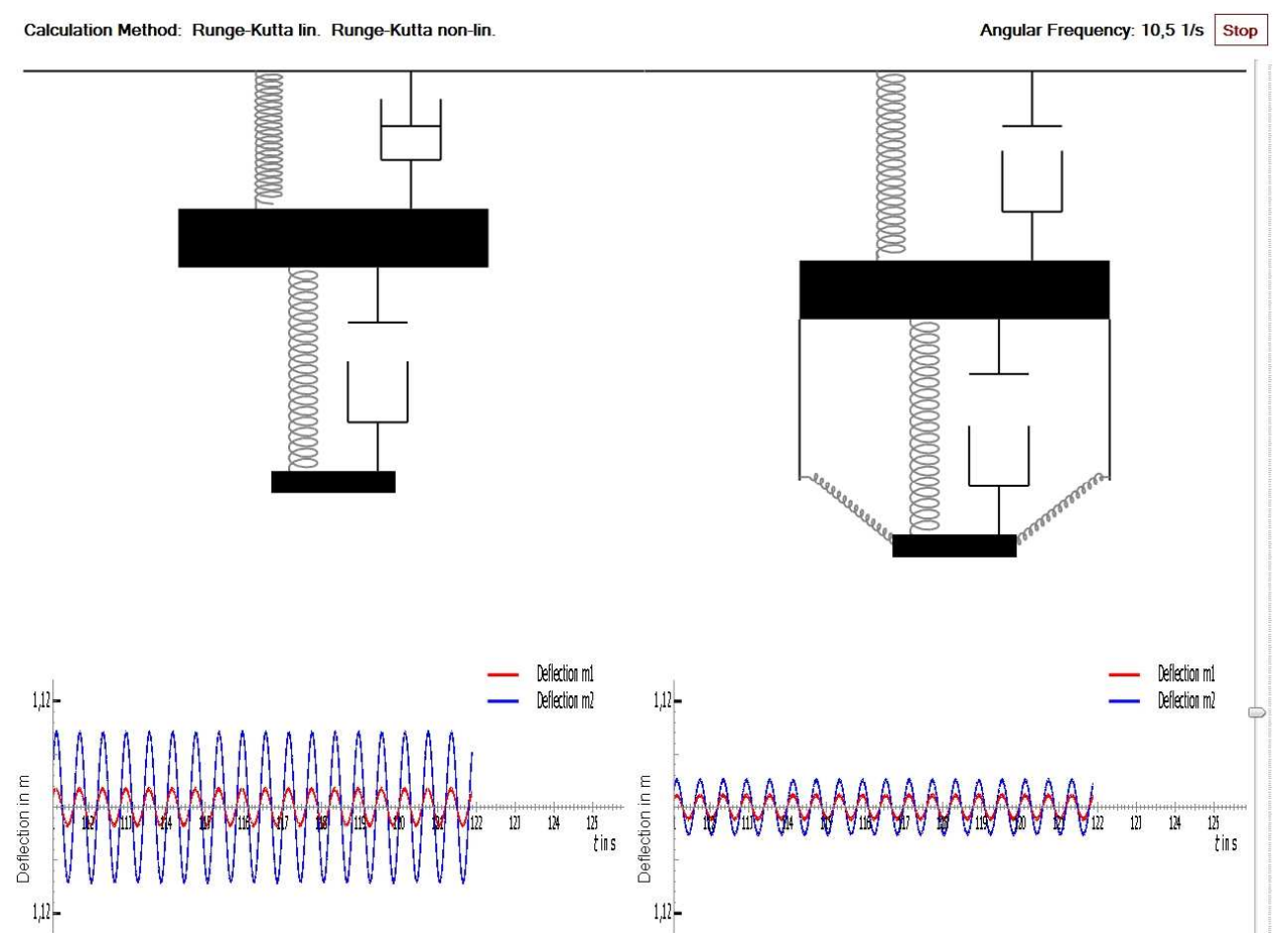

Figure 12: Comparison of linear and optimized nonlinear vibration absorber $\left(\omega / \omega_{\mathrm{c}}=0.7\right)$

In Figures 13 and 14 all parameters of the linear and the nonlinear system keep unchanged. The only difference is given by the additional (preloaded) horizontal springs with a stiffness of about $10 \%$ of the absorber spring stiffness. Thus, the nonlinear system is not optimized and as a consequence unwanted vibrations are introduced in the system [15]-[17].

The phase portrait of the linear tuned-mass damper in Figure 13 approaches a limit cycle within a short period of time. In the phase portrait of the nonlinear damper, however, temporarily two driving cycles are needed for the trajectory to be closed, a phenomenon called period doubling bifurcation [18]. 


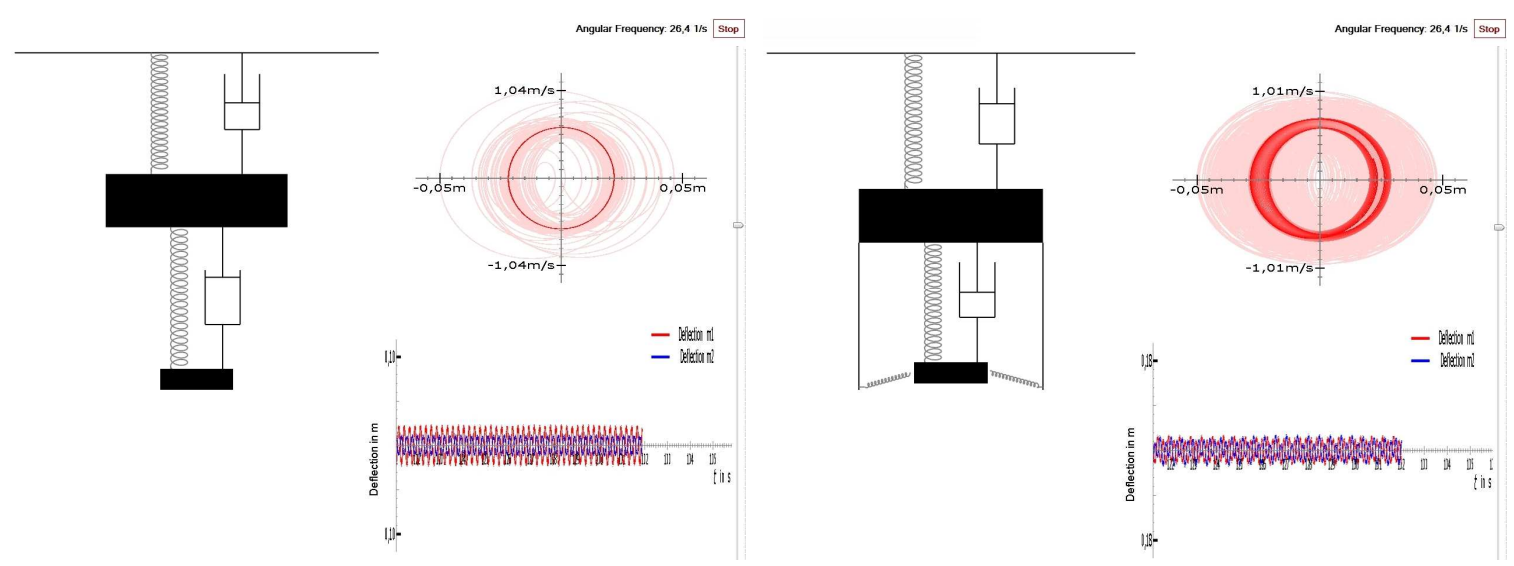

Figure 13: Comparison of linear and nonlinear vibration absorber $\left(\omega / \omega_{\mathrm{c}}=1.76\right)$

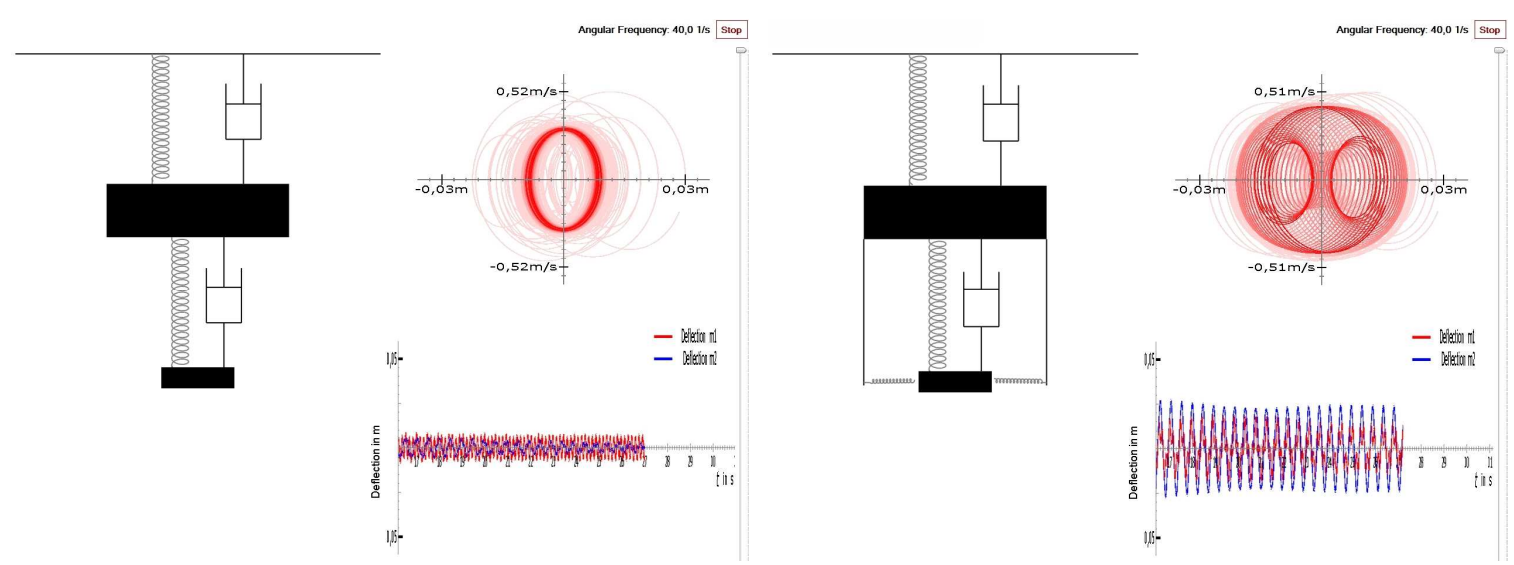

Figure 14: Comparison of linear and nonlinear vibration absorber $\left(\omega / \omega_{\mathrm{c}}=2.67\right)$

In Figure 15 the system is excited at the resonance angular frequency of the undamped primary mass-spring system $(\omega=15 \mathrm{rad} / \mathrm{s})$. The Figure shows, from left to right, the behavior of the linear vibration absorber, the optimized nonlinear absorber, and the nonlinear absorber with the parameters used in Figures 13 and 14, respectively.

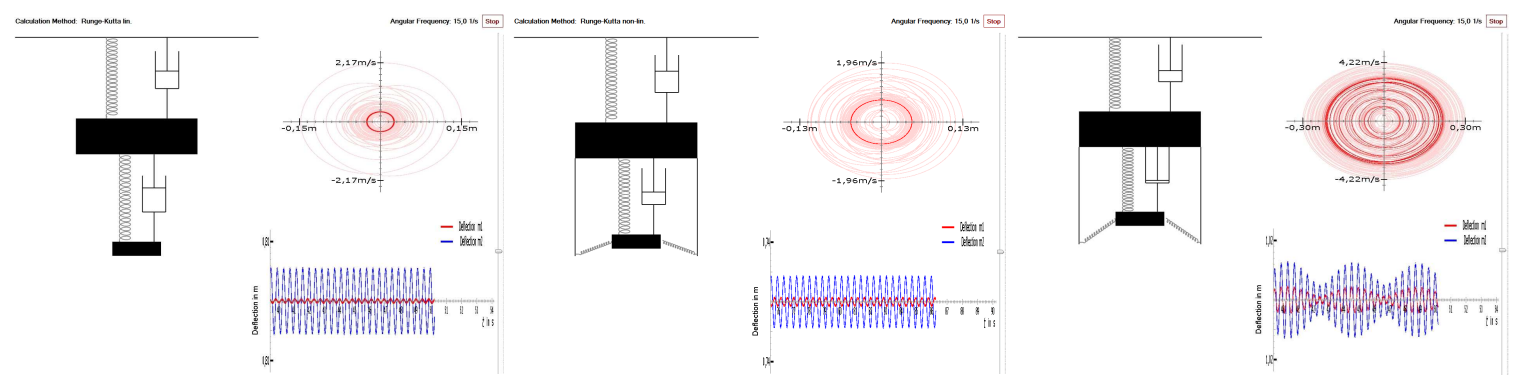

Figure 15: Comparison of linear and nonlinear vibration absorber $\left(\omega / \omega_{\mathrm{c}}=1\right)$. The right nonlinear absorber is not optimized and shows beat-like behavior and a cascade of period doublings.

In the case of the rightmost picture in Figure 15, the nonlinearity of the system leads to beatlike behavior and a cascade of period doublings in the phase portrait.

Due to damping and in the case of chaotic behavior, the phase portrait will be smeared out to unrecognizability for $t \rightarrow \infty$ (see Figures 13 and 15). For this reason, Poincaré sections have been implemented in the programs. The basic idea of Poincaré sections (or "return maps") is to plot the phase portraits only at those discrete points in time that are integral multiples of the 
excitation period $T$, as depicted by the red dots in Figure 16. In the case of a linear system the corresponding trajectory in the phase space returns, after the transient motion decays, periodically to its initial position and forms a closed curve, the limit cycle (Figure 13, left). In nonlinear systems it is possible that the trajectories smear out the available phase space (Figure 13, right), indicating the unpredictability of the system's behavior. Hence, in the stationary state of the linear system the phase space trajectory returns to the same position after every cycle, while nonlinear systems can produce clouds of irregularly distributed points that cluster in limited domains of the phase space and possess topologically highly complex fractal structures [18].

In Figure 16 Poincaré sections of the primary mass phase spaces of a nonlinear dynamic vibration absorber system are depicted for a series of excitation angular frequencies. The parameters of the (not optimized) nonlinear absorber of Figures 13 and 14 are used, and the distribution of the points resembles strikingly that one of a strange attractor.
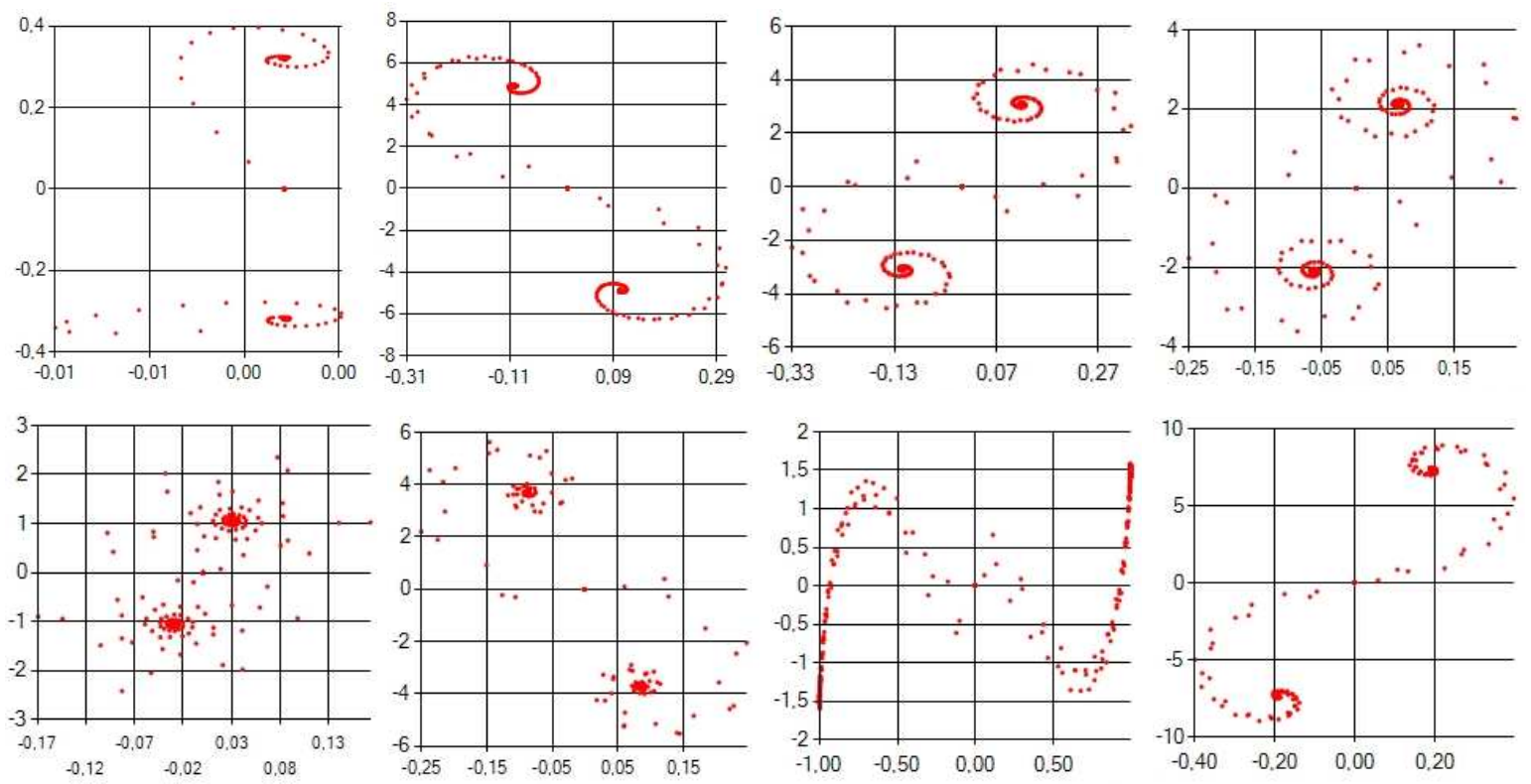

Figure 16: Primary mass Poincaré sections for $\omega=5.9,11.7,12.4,12.6,13.0,16.6,17.2$, and $17.7 \mathrm{rad} / \mathrm{s}$ (from top left to bottom right; same parameters as in Figures 11 and 12)

Furthermore, both programs provide the opportunity to choose an integration method for the numerical solution of Equations (10), see Figure 17.
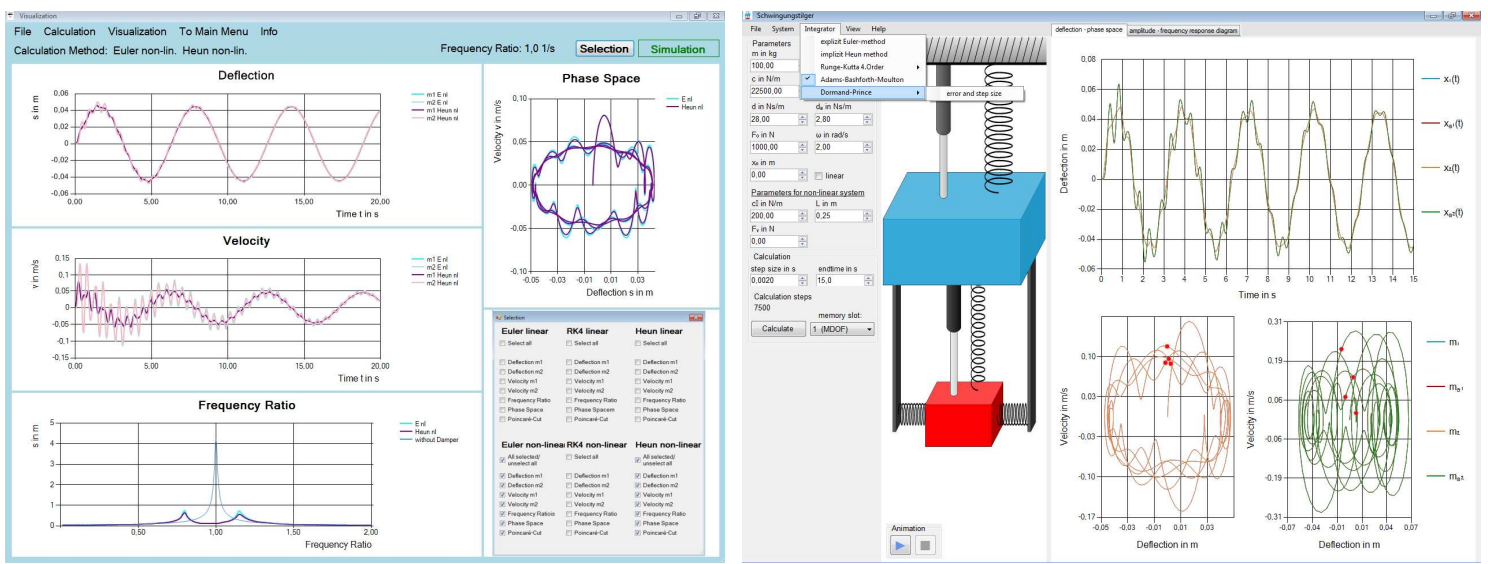

Figure 17: Examples for the comparison of different integration methods, as implemented by the two project groups (group A left: Euler and Heun, group B right: RK4 and Adams-Bashforth-Moulton) 
Five different options with different orders of truncation errors are available, namely three explicit as well as one implicit one-step methods and a multistep predictor-corrector method (Adams-Bashforth-Moulton). Both programs allow the comparison of the simulation results for the different integration methods in the same graphs (Figure 17).

The Dormand-Prince method, implemented by group B, is a member of the Runge-Kutta family and uses both a fourth- and a fifth-order solution. The difference between these solutions can be used as an error estimator for adaptive stepsize control. In Figure 18 the interplay between error per computation step and stepsize adaption is depicted. In the left figure a predefined maximum error of $10^{-3}$ was chosen, which resulted in 177 computation steps for a 15 second simulation of a linear tuned-mass damper system. A reduction of the maximum error to $10^{-5}$ took 436 computation steps, and an error limit of $10^{-8}$, as represented in the right figure, led to 2590 computation steps.
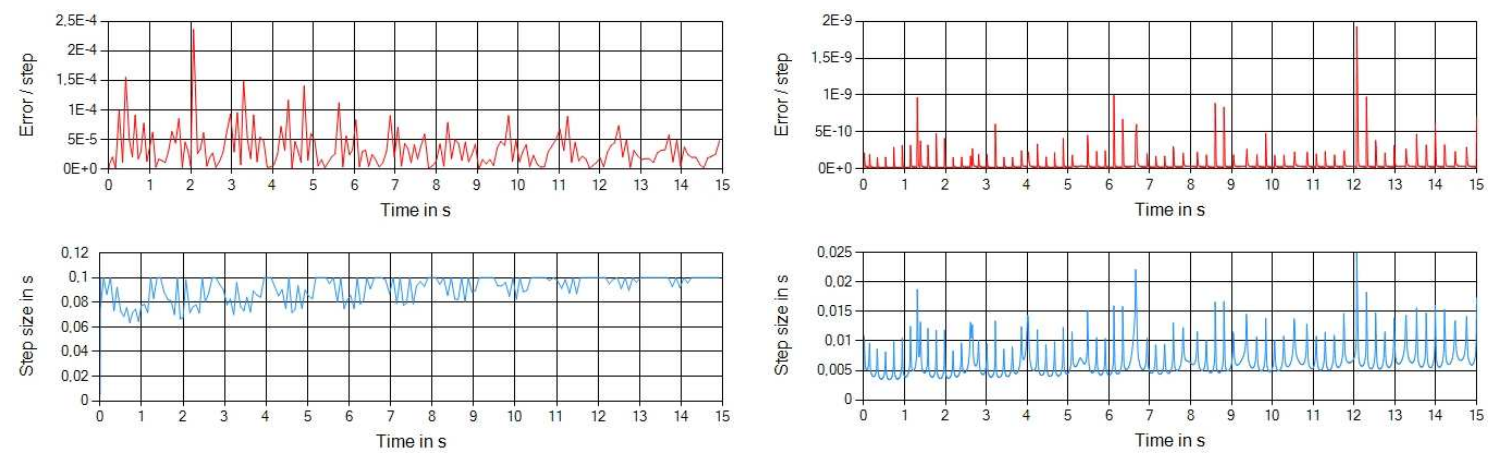

Figure 18: Adaptive stepsize control for the Dormand-Prince method with predefined maximum errors per step of $10^{-3}$ (left) and $10^{-8}$ (right); the error/step is plotted red and the stepsize blue.

The respective computation times of the various integration methods for the chosen maximum error per step are displayed in the left bottom edge of group B's graphical user interface (see Figures 8 and 9).

\section{Summary and Conclusions}

Driven by the new demands of $21^{\text {st }}$ century engineering education, we have implemented a project-based learning environment in our undergraduate engineering degree program that is characterized by active, team-oriented learning, interdisciplinary teaching, introduction of mathematical and scientific concepts in the context of application, and a faculty devoted to developing future engineers as mentors and coaches.

Multidisciplinary software projects supplemental and complementary to the lectures are part of the curriculum in the early phases of the undergraduate degree program. The software project discussed in this paper was the numerical simulation, visualization and analysis of the dynamics of tuned-mass dampers. In addition to linear mass-spring-damper absorbers, a nonlinearity of the Duffing type was introduced by a perpendicular spring configuration, which increases the absorber's effective bandwidth. Such nonlinearities can affect chaotic behavior of the system for a certain choice of parameters and are therefore an interesting and worthy object of study.

The outcome of the project was rewarding with a convincing effort to performance ratio. The student-developed programs enable the simulation of linear and nonlinear tuned-mass dampers within a variety of parameter configurations with effortless ease. In addition, it allows a gradual approach to the parameter configuration necessary for the appearance of chaotic behavior. 
The dynamic visual output of these programs can increase and enhance the understanding of various phenomena of vibration absorption; they provide a playful insight into the interaction of primary and absorber mass and are therefore well suited as a teaching aid. Both programs introduced in this paper can be downloaded, free of charge, from the website https://fahrzeugtechnik.fh-joanneum.at/software/VibrationAbsorber/, and can be installed and executed on computers running the Windows operating system.

Projects of this kind, complementary to the mathematics, informatics and science lectures, comprise the opportunity to show the students quite plainly the value of the just learned methods and algorithms. In this way, their attentiveness and their appreciation for the newly learnt topics are increased and they are given the chance to look way beyond the standard curriculum of engineering education. By assigning ambitious tasks that can only be accomplished in cooperation we equip our future graduates with stronger skills in communication, teamwork and knowledge integration in addition to sound technical competence.

\section{Acknowledgments}

Two of the authors (G.B. and C.S.) would like to express their sincere gratitude to all participating students for their high motivation and excellent performance.

\section{Bibliography}

[1] National Science Foundation, The Action Agenda for Systemic Engineering Education Reform, NSF 98-27, Arlington, VA (1998)

[2] John W. Prados, Engineering Education in the United States: Past, Present, and Future, International Conference on Engineering Education (ICEE-98), Rio de Janeiro, Brazil (1998)

[3] W. E. Kastenberg, G. Hauser-Kastenberg, and D. Norris, An Approach to Undergraduate Engineering Education for the $21^{s t}$ Century, $36^{\text {th }}$ ASEE/IEEE Frontiers in Education Conference, San Diego, CA (2006)

[4] E. Bratschitsch, A. Casey, G. Bischof, and D. Rubeša, 3-phase multi subject project-based learning as a didactical method in automotive engineering studies, Proceedings of the ASEE Annual Conference, Honolulu, HI (2007)

[5] SAE International, Warrendale (PA), https://www.fsaeonline.com/, retrieved January 28, 2020

[6] H. Frahm, Device for Damping Vibrations of Bodies, U.S. Patent 989,958, Apr. 18, 1911.

[7] G. Bischof, A. Zwölfer, and D. Rubeša, Correlation Between Engineering Students' Performance in Mathematics and Academic Success, Proceedings of the ASEE Annual Conference, Seattle, WA (2015)

[8] E. Kreyszig, Advanced Engineering Mathematics, John Wiley \& Sons, $9^{\text {th }}$ Edition (2005)

[9] G. Bischof, E. Bratschitsch, A. Casey, and D. Rubeša, Facilitating engineering mathematics education by multidisciplinary projects, Proceedings of the ASEE Annual Conference, Honolulu, HI (2007)

[10] J. P. Den Hartog, Mechanical Vibrations, McGraw-Hill Book Company (1934, republished by Dover Publications, NY, 2013).

[11] K. Liu, and G. Coppola, Optimal design of damped dynamic vibration absorber for damped primary systems, Transactions of the Canadian Society for Mechanical Engineering, Vol. 34, No. 1 (2010)

[12] A. F. Vakakis, O. Gendelman, L. A. Bergman, D. M. McFarland, G. Kerschen, Y. S. Lee, Nonlinear Targeted Energy Transfer in Mechanical and Structural Systems, Springer, Series: Solid Mechanics and Its Applications, 2009.

[13] G. Habib, T. Detroux, R. Viguié, and G. Kerschen, Nonlinear generalization of Den Hartog's equal-peak method, Mech. Syst. Signal Process. 52-53 (2015)

[14] T. Detroux, G. Habib, L. Masset, and G. Kerschen, Performance, robustness and sensitivity analysis of the nonlinear tuned vibration absorber, Mech. Syst. Signal Process. 60-61 (2015)

[15] A. S. Alsuwaiyan, Design and Analysis of a Simple Nonlinear Vibration Absorber, IOSR-JMCE, Vol. 11, Issue 2 Ver. VI (2014)

[16] H. J. Rice, Combinational instability of the nonlinear vibration absorber, Journal of Sound and Vibrations, 108,4 (1986)

[17] J. Shaw, S. W. Shaw, and A. G. Haddow, On the response of the nonlinear vibration absorber, Journal of Nonlinear Mechanics, 24 (1989)

[18] J. Argyris, G. Faust, M. Haase, and R. Friedrich, An Exploration of Dynamical Systems and Chaos, Springer Berlin-Heidelberg, $2^{\text {nd }}$ edition (2015) 